\title{
Liquid-Liquid Two-Phase Flows in Small Channels and the Effects of Polymeric Additives
}

\author{
Panagiota Angeli \\ ThAMeS Multiphase, Department of Chemical Engineering, University College London \\ Torrington Place, London WC1E 7JE, United Kingdom \\ p.angeli@ucl.ac.uk
}

Two phase liquid-liquid flows in microfluidic devices have found several chemical engineering applications in, for example, mixing, separations and reactions. From the various patterns that can form, plug flow is particularly favoured because it offers increased mixing and large interfacial areas. However, most of the studies consider plug flow in Newtonian liquids with low viscosities while many fluids for industrial applications are likely to exhibit complex rheological behaviour.

The talk will present recent studies on the hydrodynamic characteristics and velocity fields of aqueous-organic twophase flows in small channels. Xanthan gum solutions in water at different concentrations were used as the aqueous phase while the organic phase was a silicon oil. For the studies, two innovative micro Particle Image Velocimetry ( $\mu$ PIV) techniques were developed, namely high speed bright field $\mu \mathrm{PIV}$, and two-colour $\mu \mathrm{PIV}$ which allows the simultaneous capture of the velocity profiles in both phases. The results revealed a profound effect of the polymer on the flow pattern transitions in the small channels and on the geometric characteristics of the plug regime in paricular. These changes will be discussed in relation to the shear rate and viscosity fields which develop in the non Newtonian aqueous phases. 